Article

\title{
Fabrication of Self-Healable Magnetic Nanocomposites via Diels-Alder Click Chemistry
}

\author{
Yi-Huan Lee ${ }^{1,2, *}$, Yan-Nian Zhuang ${ }^{1}$, Hsin-Ta Wang ${ }^{1}{ }^{\circledR}$, Ming-Feng Wei ${ }^{3,4}$, Wen-Chi Ko ${ }^{1}$, \\ Wei-Jen Chang ${ }^{5,6, *}$, Tun-Fun Way ${ }^{2}$ and Syang-Peng Rwei ${ }^{1,2}$ (D) \\ 1 Department of Molecular Science and Engineering, National Taipei University of Technology, \\ Taipei 10608, Taiwan; nickhamesome@gmail.com (Y.-N.Z.); htwang@ntut.edu.tw (H.-T.W.); \\ asd14120asd@gmail.com (W.-C.K.); f10714@ntut.edu.tw (S.-P.R.) \\ 2 Research and Development Center for Smart Textile Technology, National Taipei University of Technology, \\ Taipei 10608, Taiwan; tfway1951@gmail.com \\ 3 Graduate Institute of Oncology, College of Medicine, National Taiwan University, Taipei 10048, Taiwan; \\ wiselyway@gmail.com \\ 4 National Taiwan University Cancer Center, College of Medicine, National Taiwan University, \\ Taipei 10048, Taiwan \\ 5 School of Dentistry, College of Oral Medicine, Taipei Medical University, Taipei 11031, Taiwan \\ 6 Dental Department, Taipei Medical University Shuang-Ho Hospital, New Taipei City 23561, Taiwan \\ * Correspondence: yihuanlee@mail.ntut.edu.tw (Y.-H.L.); cweijen1@tmu.edu.tw (W.-J.C.); \\ Tel.: +886-2-27712171 (ext. 2410) (Y.-H.L.); +886-2-27361661 (ext. 5148) (W.-J.C.)
}

Received: 11 December 2018; Accepted: 27 January 2019; Published: 1 February 2019

\begin{abstract}
In this study, we report a novel approach to fabricate an organic/inorganic magnetic hybrid system capable of self-healing, wherein a polycaprolactone-poly(furfuryl glycidyl ether) copolymer (PCLF) serving as the structure template was first synthesized, followed by the incorporation of iron oxide nanoparticles-decorated multiwalled carbon nanotubes (IONPs-MWCNTs) and 1,1'-(methylenedi-4,1-phenylene)bismaleimide (BMI) into the polymer matrix to form a covalently crosslinked hybrid network via a Diels-Alder (DA) reaction. For this system, the reactive combination of diene and dienophile from furan/maleimide, MWCNT/furan, and MWCNT/maleimide could facilely induce multiple DA reactions that imparted a versatile route to efficiently introduce IONPs-MWCNTs into the organic polymer hosts, resulting in a uniform distribution of IONPs-MWCNTs that led to a hybrid system with superparamagnetic properties. Beside the magnetic behavior, such material synergistically exhibited a superior ability for healing scratch defects via a retro-DA reaction. Therefore, this crosslinked PCLF/BMI/IONPs-MWCNTs hybrid system which exhibits multifunctional properties including superparamagnetic behavior and self-repairability can serve as an intelligent material for developing advanced electromagnetic applications.
\end{abstract}

Keywords: self-healing; Diels-Alder reaction; nanocomposites; copolymer; MWCNT; iron oxide nanoparticles

\section{Introduction}

The concept of smart materials, such as substances which self-heal without foreign mediation, is a promising substitute to material design that is insensitive to defects to sustain safety and/or performance. Such systems have the capability to heal themselves without outside mediation and restore their performance by using the properties intrinsically installed within them. So far, several approaches for the fabrication of autonomous self-healing materials, such as microscale vascular [1,2], capsule [3,4], and reservoir [5,6] systems, have been established. For these materials, the effective 
self-healing process often resorts to the release of an active ingredient which could be catalyst, monomer or crosslinking agent at the crack tip to efficiently block the crack advancement [7]. However, the irreversible autonomous systems often provide the self-repair function only once because of the consumption of the limited healing agents, leading to a potential disadvantage for their long-term use $[8,9]$. Therefore, the development of an efficient and versatile strategy for self-repairing materials capable of healing repeated damage events is greatly required to overcome this drawback from the irreversible systems.

Recently, there is a growing attention on reversible self-healing systems. These self-healing polymers with inherently reversible interactions are capable of undergoing infinite break and healing cycles upon outside stimulations, and thus can sufficiently provide high self-healing proficiency to rebuild the damaged area at the same location. A number of strategies have been built up to develop reversible self-healing systems by utilizing chemical reactions including cycloaddition [10], metathesis reaction [11,12], metal-ligand complexation [13], ring-opening [14], as well as complexation/decomplexation reactions $[15,16]$. Among them, the Diels-Alder (DA) reaction was often recognized as one of the most favorable reaction types applied for reversible self-repair systems since this click reaction can be easily triggered by a thermal treatment. For example, furan and maleimide serving respectively as electron-rich and electron-deficient moiety undergo a thermally reversible cycloaddition reaction in the temperatures range from 100 to $150{ }^{\circ} \mathrm{C}$, which is suitable for daily applications [17-25]. Despite the continuous progress of the DA systems, it should be noted that most studies focused on their synthetic routes and self-healing abilities. However, specific efforts with the DA based organic/inorganic hybrid systems are less common. In principle, heat activated self-repair polymers are superior matrix materials used in hybrid systems. The incorporation of inorganic nanomaterials for the fabrication of hybrid materials would further offer multifunctional properties that create expanded opportunities for developing advanced applications. For example, self-healing nanohybrids based on DA reactions with the incorporation of MWCNTs (multiwalled carbon nanotubes), GOs (graphene oxide), or inorganic nanoparticles showed potential for practical use [26-32]. In particular, carbon nanotubes have received a great interest due to their superior electrical, thermal, and mechanical properties compared to those of traditional fiber materials [33,34]. The decoration of carbon nanotubes with magnetic nanoparticles, such as iron oxides, can further enhance or diversify the optical, electromagnetic, and electrochemical properties of the carbon nanotubes $[35,36]$. The magnetic nanotubes-based hybrid systems can be used in various applications, e.g., electrochemical sensing, rechargeable batteries, and electromagnetic interference shielding [37-40]. Developing novel carbon nanotubes/iron oxides materials with self-repairability is highly desirable to improve the long-term durability of these application fields.

In this study, a novel self-healing hybrid material system was successfully developed by incorporating iron oxide nanoparticles-multiwalled carbon nanotubes (IONPs-MWCNTs) into a crosslinked PCL-based network. The IONPs-MWCNTs were first synthesized by an "outside-decorating" method to connect IONPs to the surfaces of the MWCNTs, followed by a mixing process to combine the IONPs /MWCNTs with 1,1'-(methylenedi-4,1-phenylene)bismaleimide (BMI) and polycaprolactone-poly(furfuryl glycidyl ether) copolymer (PCLF) which served as a polymeric matrix for the hybrid system. This material could be reversibly crosslinked via DA/retro DA chemistry and exhibit multiple self-healing cycles. Therefore, even complex damages and serious cracks could be efficiently recovered. More importantly, these nanocomposites also exhibited superparamagnetic properties. Thus, the organic/inorganic hybrid material system developed in the current study can be used as a novel material for future electromagnetic applications. 


\section{Materials and Methods}

\subsection{Materials}

$\varepsilon$-Caprolactone (CL) purchased from Sigma-Aldrich (St. Louis, MO, USA) was dried over calcium hydride $\left(\mathrm{CaH}_{2}\right)$, followed by freeze-pump-thaw cycles and a distillation under a reduced pressure. Furfuryl glycidyl ether (FGE) purchased from Sigma-Aldrich were used as received. Poly(caprolactone)diol (PCL diol) was purchased from Sigma-Aldrich and purified by recrystallization in methanol. MWCNTs with diameters in the range of 10-20 nm and with lengths about 5-15 $\mu \mathrm{m}$ were purchased from Conjutek Co., Ltd. (Taipei, Taiwan). 1,5,7-Triazabicyclo[4.4.0]dec-5-ene (TBD, 98\%, Fluka, Morristown, NJ, USA), iron (II) chloride tetrahydrate $\left(\mathrm{FeCl}_{2} \cdot 4 \mathrm{H}_{2} \mathrm{O}, 98 \%\right.$, Sigma-Aldrich), iron (III) chloride hexahydrate $\left(\mathrm{FeCl}_{3} \cdot 6 \mathrm{H}_{2} \mathrm{O}, 98 \%\right.$, Sigma-Aldrich) and 1,1'-(methylenedi-4,1-phenylene)bismaleimide (95\%, Sigma-Aldrich) were used without further purification. Additionally, all other reagents and solvents were used as received without any purification.

\subsection{Methods}

\subsubsection{Synthesis of Polycaprolactone-poly(furfuryl glycidyl ether) Copolymers (PCLF)}

The synthesis procedure of the PCLF copolymer sample is shown in Figure 1. First, PCL diol was added into a flask (flask A) charged with dried nitrogen. TBD was then added into the flask, and the mixture was subsequently stirred at room temperature for $2 \mathrm{~h}$. Meanwhile, CL and FGE monomers were added into another flask (flask B) and stirred in dried nitrogen at room temperature. After 30 min of mixing, the monomers in flask B were then transferred into flask A. The resulting mixture was then stirred at $110{ }^{\circ} \mathrm{C}$ to carry out the ring-opening polymerization to form PCLF copolymer. The copolymerization was allowed to progress at $110^{\circ} \mathrm{C}$. After $6 \mathrm{~h}$ of reaction, methanol was added into the reaction system to terminate the polymerization. The mixture solution was further concentrated and precipitated into ethanol medium. Finally, the purified PCLF powders were obtained by removing the solvent and then stored in a vacuum oven.
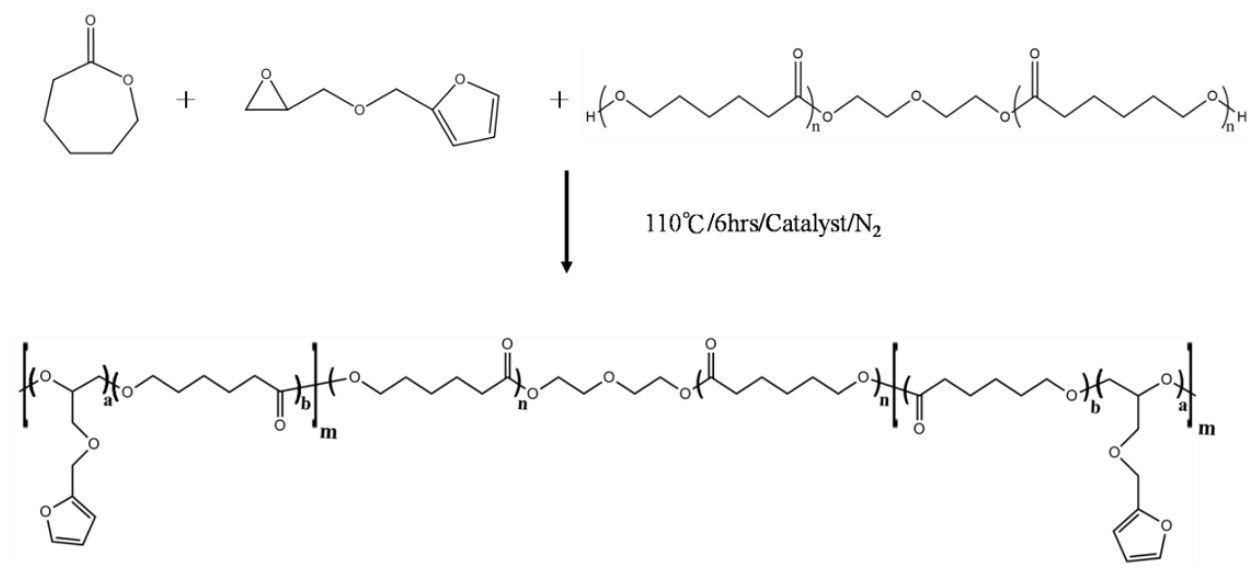

Figure 1. Synthetic route of the polycaprolactone-poly(furfuryl glycidyl ether) copolymer (PCLF) copolymer system.

\subsubsection{Synthesis of IONPs-MWCNTs Hybrid}

The synthesis of IONPs-MWCNTs was carried out by using the co-precipitation method [41-43]. MWCNTs were first treated by using a binary acid mixture of concentrated $\mathrm{H}_{2} \mathrm{SO}_{4}$ and $\mathrm{HNO}_{3}$ (volume ratio $=3: 1$ ) at $90{ }^{\circ} \mathrm{C}$ for $1.5 \mathrm{~h}$, followed by washing them with distilled water. Until the $\mathrm{pH}$ value of the MWCNTs solution reached 7, the MWCNTs were dried by removing residual solvent in a vacuum oven. In the next stage, the dried MWCNTs were dispersed in distilled water by using ultra-sonication. 
Subsequently, $\mathrm{FeCl}_{2} \cdot 4 \mathrm{H}_{2} \mathrm{O}$ and $\mathrm{FeCl}_{3} \cdot 6 \mathrm{H}_{2} \mathrm{O}$ precursors were added into the MWCNTs solution system and then stirred in dry nitrogen at room temperature. After complete mixing, an ammonia solution ( $30 \mathrm{vol} / \mathrm{vol} \%$ ) was slowly added into the MWCNTs-based mixture until the $\mathrm{pH}$ value of the solution reached the range of about 10-11. The reaction system was then heated at $80{ }^{\circ} \mathrm{C}$ for $2 \mathrm{~h}$ to in-situ synthesize the IONPs-MWCNT hybrid. Finally, the obtained products were further purified by washing them with distilled water, followed by drying in a vacuum oven at $50{ }^{\circ} \mathrm{C}$ for $24 \mathrm{~h}$.

\subsubsection{Preparation of Crosslinked PCLF/BMI/IONPs-MWCNTs Hybrid Material}

After the successful synthesis of IONPs-MWCNTs, the inorganic hybrid material was further added to PCLF/THF solutions in three different weight fractions of IONPs-MWCNTs to PCLF of $2 \mathrm{wt} \%, 5 \mathrm{wt} \%$, and $10 \mathrm{wt} \%$. These mixtures were subjected to ultrasonic disruption for at least $12 \mathrm{~h}$. After drying, 1,1'-(methylenedi-4,1-phenylene)bismaleimide with molar ratio of furan to maleimide of 1:1 was added into the PCLF/IONPs-MWCNT mixtures and stirred in bulk at $140{ }^{\circ} \mathrm{C}$ for $2 \mathrm{~h}$. The final hybrid samples were placed in a vacuum oven at $140^{\circ} \mathrm{C}$, followed by a slow decrease in temperature from $140{ }^{\circ} \mathrm{C}$ to $80{ }^{\circ} \mathrm{C}$. The temperature of the oven was then maintained at $80^{\circ} \mathrm{C}$ for $24 \mathrm{~h}$.

\subsubsection{Characterization Methods}

${ }^{1} \mathrm{H}$ NMR analysis of the PCLF copolymer was conducted by using a $400 \mathrm{MHz}$ Bruker Avance spectrometer at room temperature. $\mathrm{CDCl}_{3}$ was used as solvent for this measurement. The measurements of the molecular weight and molecular weight distribution of the synthesized PCLF were carried out by using a Viscotek TDA 305 system equipped with a refractive-index detector. THF was used as a mobile phase at an operating temperature of $35^{\circ} \mathrm{C}$. For the hybrid material system, the investigations of nanomorphologies were performed using a Hitachi H-7650 transmission electron microscopy operating at $75 \mathrm{kV}$ with a Gaten model $782 \mathrm{CCD}$ camera. Elemental analyses were performed by using an energy-dispersive X-ray spectroscopy detector (Oxford $\mathrm{x}$-act) attached to a Hitachi TM4000PLUS scanning electron microscopy. ATR-FTIR measurements were carried out on a Perkin Elmer Spectrum One spectrometer. Wide-angle X-ray scattering (WAXS) experiments of the hybrid samples were conducted at beamline 13A1 of the National Synchrotron Radiation Research Center (NSRRC) in Taiwan. The wavelength of the incident beam was set as $1.033 \AA$ for the WAXS analyses. Additionally, rectangular shape samples $(5 \mathrm{~mm} \times 5 \mathrm{~mm} \times 1 \mathrm{~mm})$ were used for the $\mathrm{X}$-ray measurements, and the detection time was set as $30 \mathrm{~min}$ for each sample. Thermogravimetric analyses (TGA) of these hybrid samples were performed by using a Hitachi STA7200 instrument. These measurements were carried out by heating the samples from $50{ }^{\circ} \mathrm{C}$ to $600{ }^{\circ} \mathrm{C}$ at a heating rate of $10^{\circ} \mathrm{C} /$ min under $\mathrm{N}_{2}$ atmosphere. A Hitachi DSC7000X differential scanning calorimetry was used to analyze the thermal properties of the hybrid materials. These samples were measured through a heating process from $-30{ }^{\circ} \mathrm{C}$ to $200{ }^{\circ} \mathrm{C}$ at a rate of $5{ }^{\circ} \mathrm{C} / \mathrm{min}$. The magnetic properties of the hybrid samples were studied using a Microsense 1660 Torque Vibratory Sample Magnetometer (VSM) system. These measurements were performed at room temperature under applied field in the range from $-10,000$ to 10,000 Oe, and started from $-10,000$ Oe with different step sizes $(-10,000 \sim-2000$ Oe: scan step $=1000$ Oe; $-2000 \sim-200$ Oe: scan step $=200$ Oe; $-200 \sim 0$ Oe: scan step $=20$ Oe). The self-healing ability of the hybrid system was studied by investigating the evolution of a crack healing under a Nikon ECLIPSE LV100N POL polarizing optical microscope equipped with a CCD camera. Rectangular shape samples of crosslinked PCLF/BMI/IONPs-MWCNTs hybrid ( $5 \mathrm{~mm} \times 5 \mathrm{~mm} \times$ $1 \mathrm{~mm}$ ) were cut by using a razor blade to form a crack on the surface of the samples. The cracked samples were heated in-line using a Linkam THMS600 hot stage to a specified temperature. As the setting temperature was achieved, the measured samples were isothermally heated for $5 \mathrm{~min}$ before investigation. Additionally, the crack evolution before and after the self-healing process was also investigated by using a Hitachi FlexSEM 1000 scanning electron microscopy. 


\section{Results and Discussion}

The ring opening polymerization (ROP) strategy has been widely used to facilely synthesize cyclic esters based polymers. In the current study, PCL diol which served as a macroinitiator was first mixed with TBD catalyst, followed by the addition of a mixture of CL and FGE monomers to synthesize PCLF copolymer with high yield. To monitor the polymerization progress of the reaction system, the molecular weight characterizations of the macroinitiator and polymer product were both analyzed by using GPC. On the basis of the GPC profiles, we could observe that the PCL diol macroinitiator exhibited a number-average molecular weight of $2600 \mathrm{~g} \mathrm{~mol}^{-1}$ and a polydispersity value of 2.04 . After the addition of CL/FGE mixing monomers, we could further observe that there was a molecular weight increase in the GPC trace from the original $2600 \mathrm{~g} \mathrm{~mol}^{-1}$ toward a higher molecular weight of $5700 \mathrm{~g} \mathrm{~mol}^{-1}$. This result clearly demonstrated the successful synthesis of the PCLF copolymer. All the molecular parameters of the polymerization system in the current study are listed in Table 1 . The molecular structure of the synthesized PCLF copolymer was further characterized by using ${ }^{1} \mathrm{H}-\mathrm{NMR}$ measurement. From the obtained spectrum shown in Figure 2, we could clearly identify the structural features of the sample. The characteristic peaks at 7.4 and $6.3 \mathrm{ppm}$ could be assigned to the protons of furan rings and the peak at 3.7-3.3 ppm could be assigned to the protons of opened moiety of FGE. Additionally, the proton signals associated with methylene groups of CL were also assigned. According to the integrated area ratio between the protons of furan groups (6.3 ppm) and the protons of methylene groups adjacent to carbonyl groups of CL (2.3 ppm), the molar fraction of FGE in the PCLF copolymer was calculated to be $\sim 15 \%$. Additionally, the complete disappearance of the hydrogen signals from methylene of $\varepsilon$-caprolactone monomers (2.6 ppm) and the epoxy methylene of FGE monomers confirmed the high conversion from the ROP reaction.

Table 1. Molecular characteristics of PCL diol and PCLF copolymer samples.

\begin{tabular}{ccccc}
\hline Sample & $\left.\mathbf{M}_{\mathbf{n}} \mathbf{( g / m o l}\right)$ & $\mathbf{M w}(\mathbf{g} / \mathbf{m o l})$ & PDI & FGE/CL Ratio $^{\mathbf{1}}$ \\
\hline PCL diol & 2600 & 5300 & 2.04 & - \\
PCLF & 5700 & 10,900 & 1.91 & 0.15 \\
\hline
\end{tabular}

${ }^{1}$ Based on the ${ }^{1} \mathrm{H}-\mathrm{NMR}$ results shown in Figure 2.
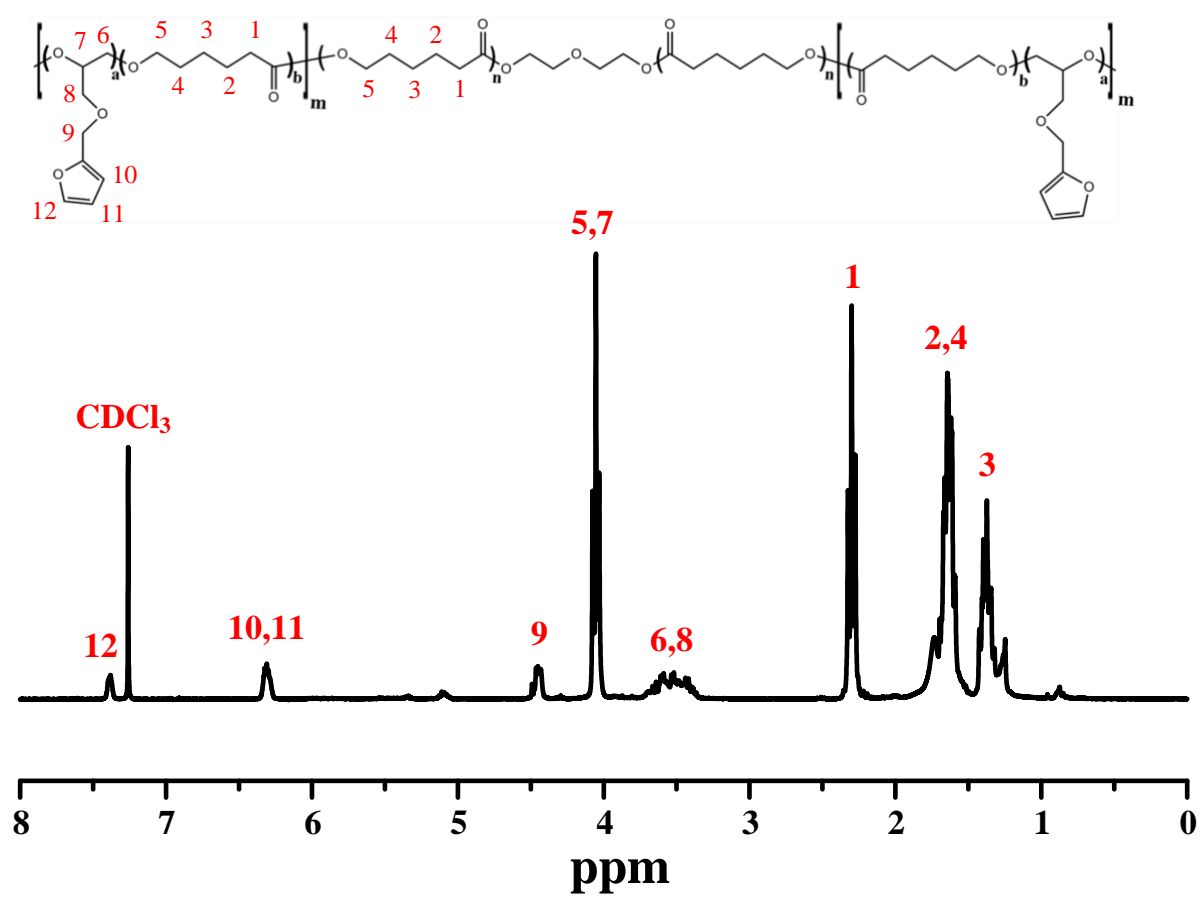

Figure 2. ${ }^{1} \mathrm{H} \mathrm{NMR}$ spectrum of the PCLF copolymer sample in $\mathrm{CDCl}_{3}$. 
In the current study, the combination of MWCNTs and monodispersed IONPs was prepared by using co-precipitation method [41-43], in which the acid-treated MWCNTs were used as structure-directing templates and the IONPs were subsequently in situ grown on the surfaces of the MWCNTs via a co-precipitation process. Figure 3a shows the TEM image of the acid-treated pristine MWCNTs prepared by drop coating MWCNTs/THF solution onto a carbon-coated copper grid. Figure $3 \mathrm{~b}$ shows the TEM image of IONPs-MWCNTs hybrid sample. It could be observed that IONPs preferentially located along the MWCNTs templates to form a one-dimensional nanofibril structured hybrid system rather than located in the empty space without the presence of MWCNTs, indicating an intimate contact between IONPs and MWCNTs that resulted from the in situ synthesis process. A higher-magnification image of the IONPs-MWCNTs hybrid sample is also shown in Figure 3c. Based on the atomic ratio between the iron and carbon signals in the EDX spectrum (Figure 3e), the loading fraction of IONPs in the IONPs-MWCNTs hybrid is found to be $\sim 56 \%$. Figure $3 \mathrm{~d}$ further shows the morphology of the PCLF/ IONPs-MWCNTs hybrid with $10 \mathrm{wt} \%$ IONPs-MWCNTs. From the TEM image, we could observe an uniform distribution of IONPs-MWCNTs in the PCLF copolymer matrix, and no serious aggregations were found. These results clearly provided the evidence that MWCNTs could interact with the furan groups of PCLF via a DA reaction, resulting in a homogeneous dispersion of IONPs-MWCNTs within the copolymer matrix. This outcome demonstrated that DA reaction could be efficiently applied to modify the surfaces of MWCNTs by using furan group as a diene. Our result also bears resemblance to that of a previous report wherein poly(furfuryl methacrylate) (PFMA) containing furfuryl functional group was covalently bonded onto the surfaces of MWCNTs via a DA reaction, thus strongly enhancing the miscibility between the MWCNTs and the polymer matrix [44]. The formation of these DA structures attached to the surfaces of MWCNTs could be confirmed by monitoring the solubility of the hybrids in solvent medium. Figure 4a shows the image of dispersion condition in DMSO for the PCLF/IONPs-MWCNTs DA adducts. We observed that the hybrids could be uniformly dispersed in DMSO solvent after a brief sonication process and the dispersity of the mixture exhibited superior dispersion stability. As the well-dispersed suspension system was heated at $160{ }^{\circ} \mathrm{C}$ for over $5 \mathrm{~h}$, the inorganic IONPs-MWCNTs gradually precipitated from the dispersion due to the occurrence of detachment of PCLF from MWCNTs surfaces via a retro-DA reaction, as shown in Figure $4 \mathrm{~b}$. In addition to the MWCNT/furan DA reaction, it has been also reported that MWCNT could serve as a "diene" and react with maleimide (dienophile) through the DA mechanism [45]. Therefore, the addition of BMI into the hybrid system to fabricate crosslinked PCLF/BMI/IONPs-MWCNTs hybrid material could provide additional support for the inorganic IONPs-MWCNTs and maintain their uniform distribution in the polymer matrix. For the crosslinked PCLF/BMI/IONPs-MWCNTs hybrid system developed in this study, a schematic illustration showing its thermo-reversible DA network formed from furan/maleimide, MWCNT/furan, and MWCNT/maleimide combinations is shown in Figure 5. Additionally, the designations and compositional characteristics of the prepared hybrid samples were tabulated in Table 2. The DA and retro-DA reactions of the PCLF/BMI/IONPs-MWCNTs hybrid system could also analyzed by using FTIR measurements (shown in Figure 6). In comparison with the characteristic profile of pristine PCLF, the spectrum of the crosslinked PCLF/BMI/IONPs-MWCNTs sample (PCLF-DA-5) exhibited additional 1773 and $1708 \mathrm{~cm}^{-1}$ peaks associated with the $C=C$ stretching of DA adducts and $C=C$ stretching vibration of BMI, respectively. This signal change clearly indicated the formation of DA adducts via a DA reaction. For the PCLF-DA-5 sample, the peak at $756 \mathrm{~cm}^{-1}$ corresponding to furfuryl moiety also disappeared, which again confirmed the occurrence of DA reaction that change the original furan rings in pristine PCLF [30]. 

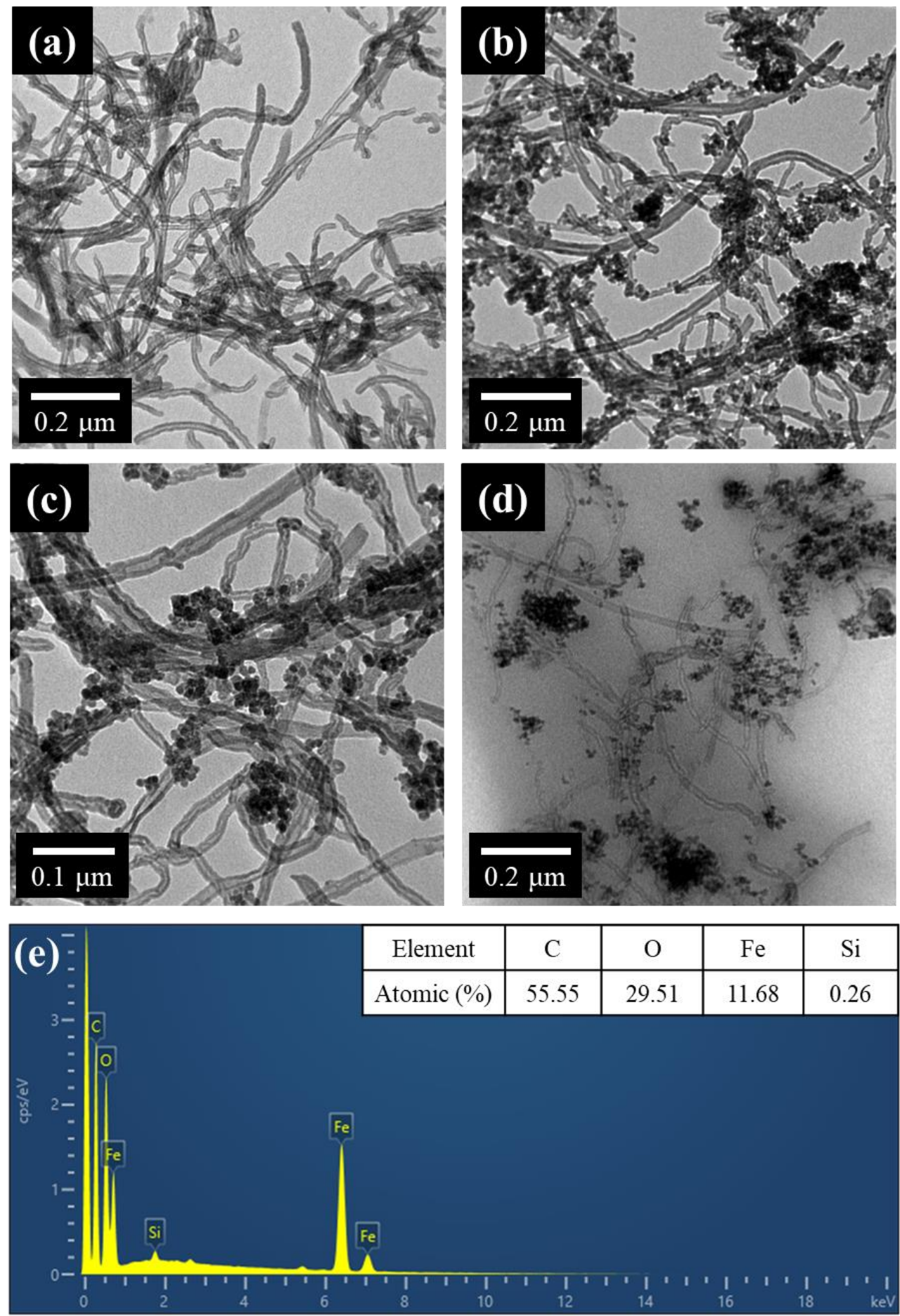

Figure 3. (a) TEM micrograph of acid-treated multiwalled carbon nanotubes (MWCNTs). (b) TEM micrograph of the iron oxide nanoparticles-multiwalled carbon nanotubes (IONPs-MWCNTs) hybrid. For this sample, its corresponding high magnification TEM image is further shown in (c). The TEM image in (d) shows the morphology of PCLF/IONPs-MWCNTs sample containing $10 \mathrm{wt} \%$ IONPs-MWCNTs, indicating a good dispersion of IONPs-MWCNTs in the polymer matrix. (e) EDX spectrum of IONPs-MWCNTs hybrid deposited on a silicon wafer. 


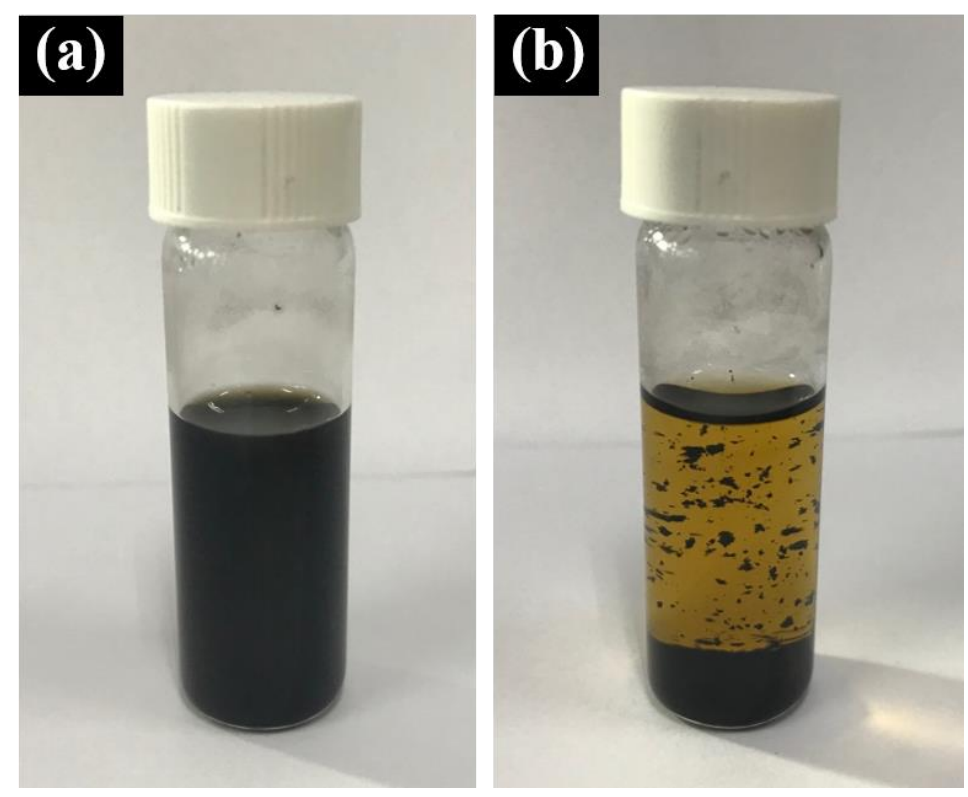

Figure 4. (a) Dispersion condition of the PCLF/IONPs-MWCNTs Diels-Alder (DA) adducts in DMSO. (b) Dispersion condition of the PCLF/IONPs-MWCNTs DA adducts in DMSO after heating at $160{ }^{\circ} \mathrm{C}$ for $5 \mathrm{~h}$.
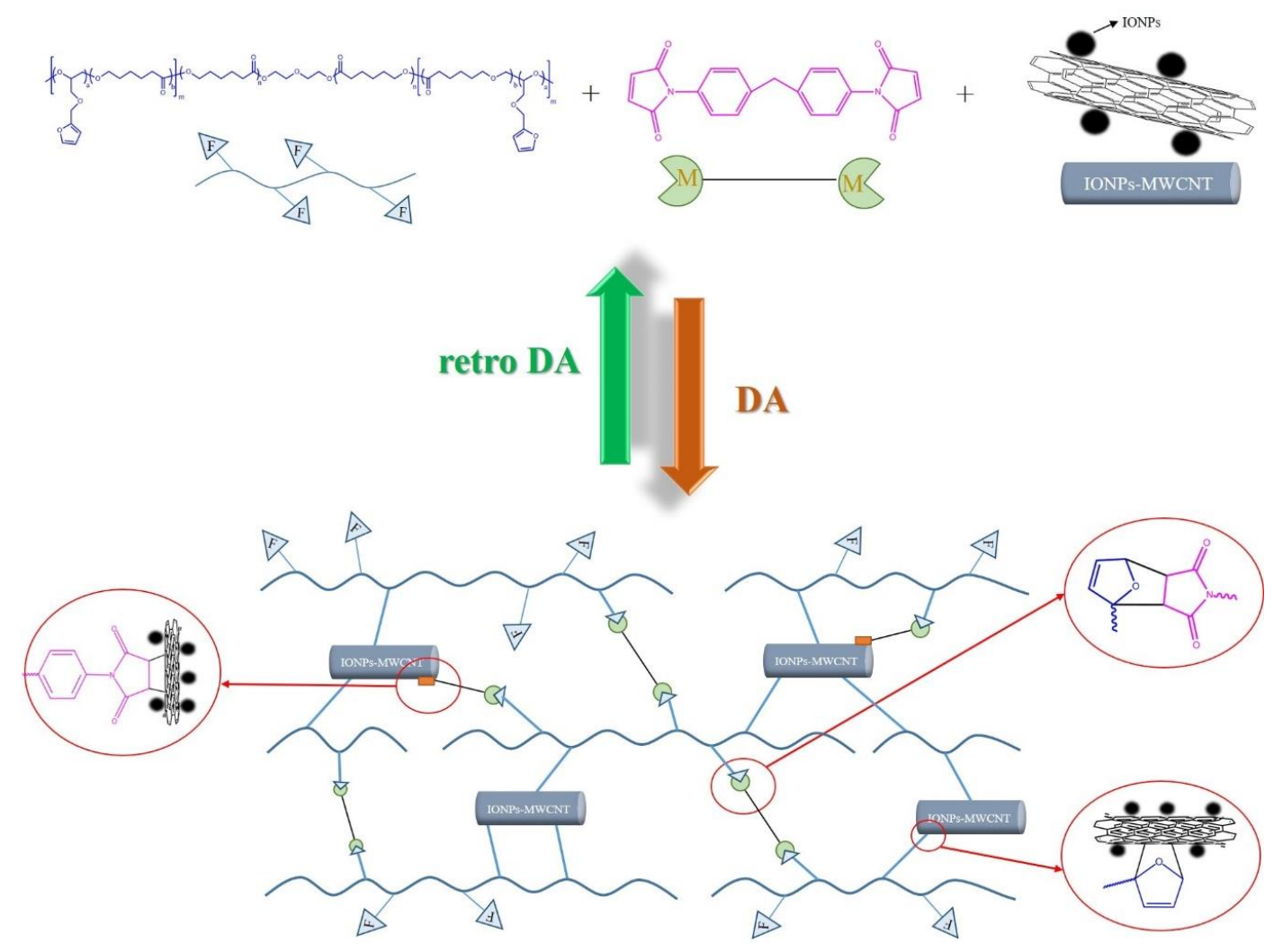

Figure 5. Schematic representation of the thermo-reversible DA network formed from furan/maleimide, MWCNT/furan, and MWCNT/maleimide combinations. 
Table 2. Composition characteristics of crosslinked PCLF/BMI/IONPs-MWCNTs hybrid samples.

\begin{tabular}{ccc}
\hline Sample $^{\mathbf{1}}$ & FGE/BMI Ratio & IONPs-MWCNTs Amount $^{2}$ \\
\hline PCLF-DA-0 & $1: 1$ & $0 \mathrm{wt} \%$ \\
PCLF-DA-2 & $1: 1$ & $2 \mathrm{wt} \%$ \\
PCLF-DA-5 & $1: 1$ & $5 \mathrm{wt} \%$ \\
PCLF-DA-10 & $1: 1$ & $10 \mathrm{wt} \%$
\end{tabular}

${ }^{1}$ Designation of crosslinked PCLF/BMI/IONPs-MWCNTs hybrid materials. ${ }^{2}$ Added ratios of IONPs-MWCNTs to PCLF copolymer.

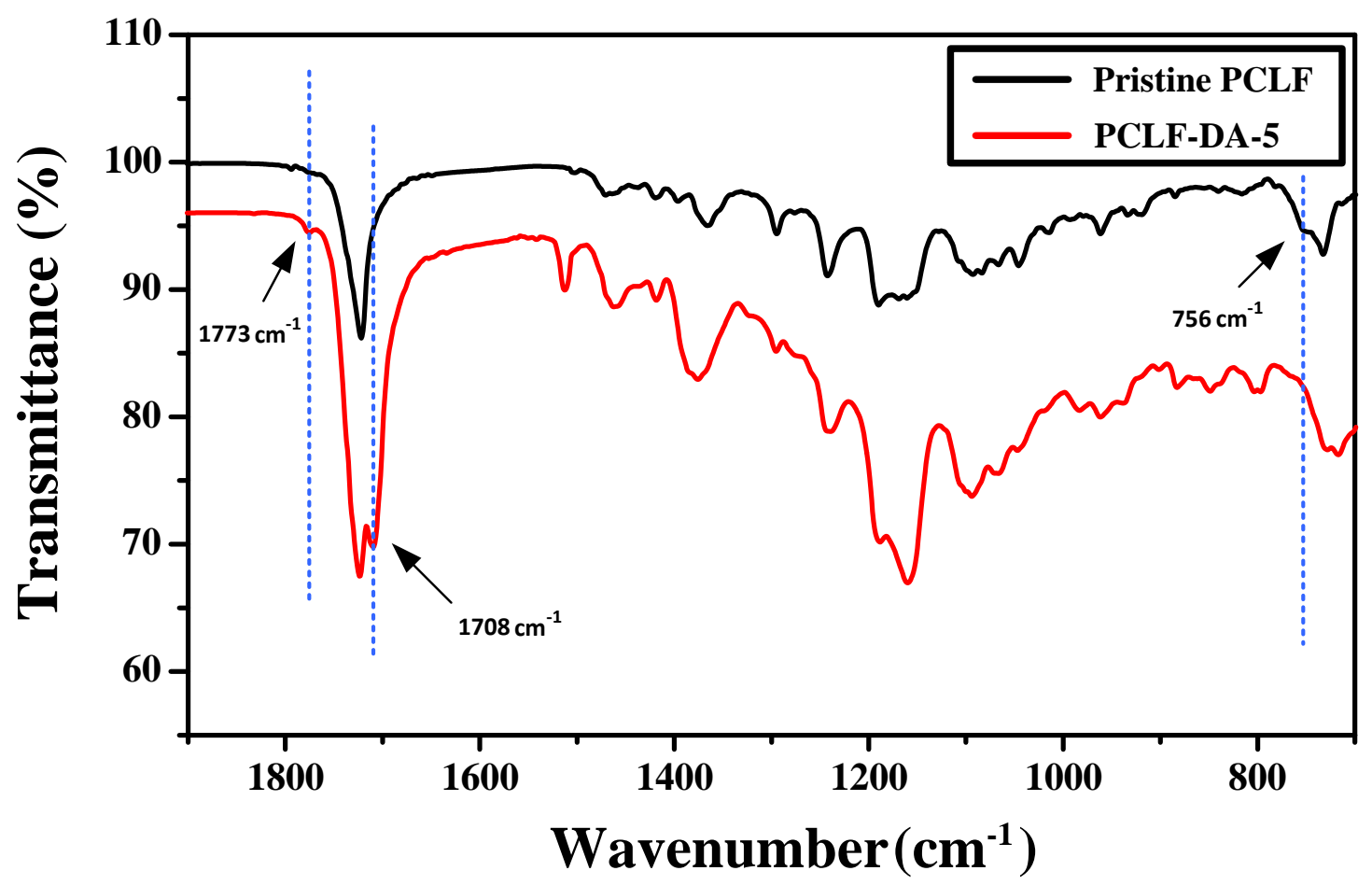

Figure 6. FTIR spectra of pristine PCLF and PCLF-DA-5 samples.

We subsequently examined the crystallography features of the hybrid system by using WAXS measurements. As shown in Figure 7, it could be observed that the WAXS profile of the PCLF-DA-0 sample revealed broad halos at $2 \theta=13.0^{\circ}$ which corresponded to the amorphous halo of PCL, indicating that the crystallization of the PCL segments was strongly suppressed by the DA crosslinked structures. In contrast to the WAXS result for crosslinked PCLF that only showed an amorphous PCL signal, the WAXS patterns of the hybrid samples consisted of a combination of the scattering characteristics of organic and inorganic parts. It could be observed that the WAXS result of the PCLF-DA-2 sample exhibited a scattering peak at $2 \theta=17.1^{\circ}$, which correspond to the crystalline plane of (002) associated with MWCNTs. Additionally, we could observe scattering peaks located at $2 \theta=$ $20.1^{\circ}, 23.5^{\circ}, 28.4^{\circ}, 35.0^{\circ}, 37.2^{\circ}$, and $40.7^{\circ}$, which corresponded to the crystallographic reflections from (220), (311), (400), (422), (511), and (440) crystalline planes of IONPs, respectively. Upon increasing the fraction of IONPs-MWCNTs, the WAXS results of the hybrid samples exhibited a progressively increasing intensity of the IONPs-MWCNTs peaks, indicating the sufficient incorporation of the inorganic nanomaterials into the polymer matrix. 


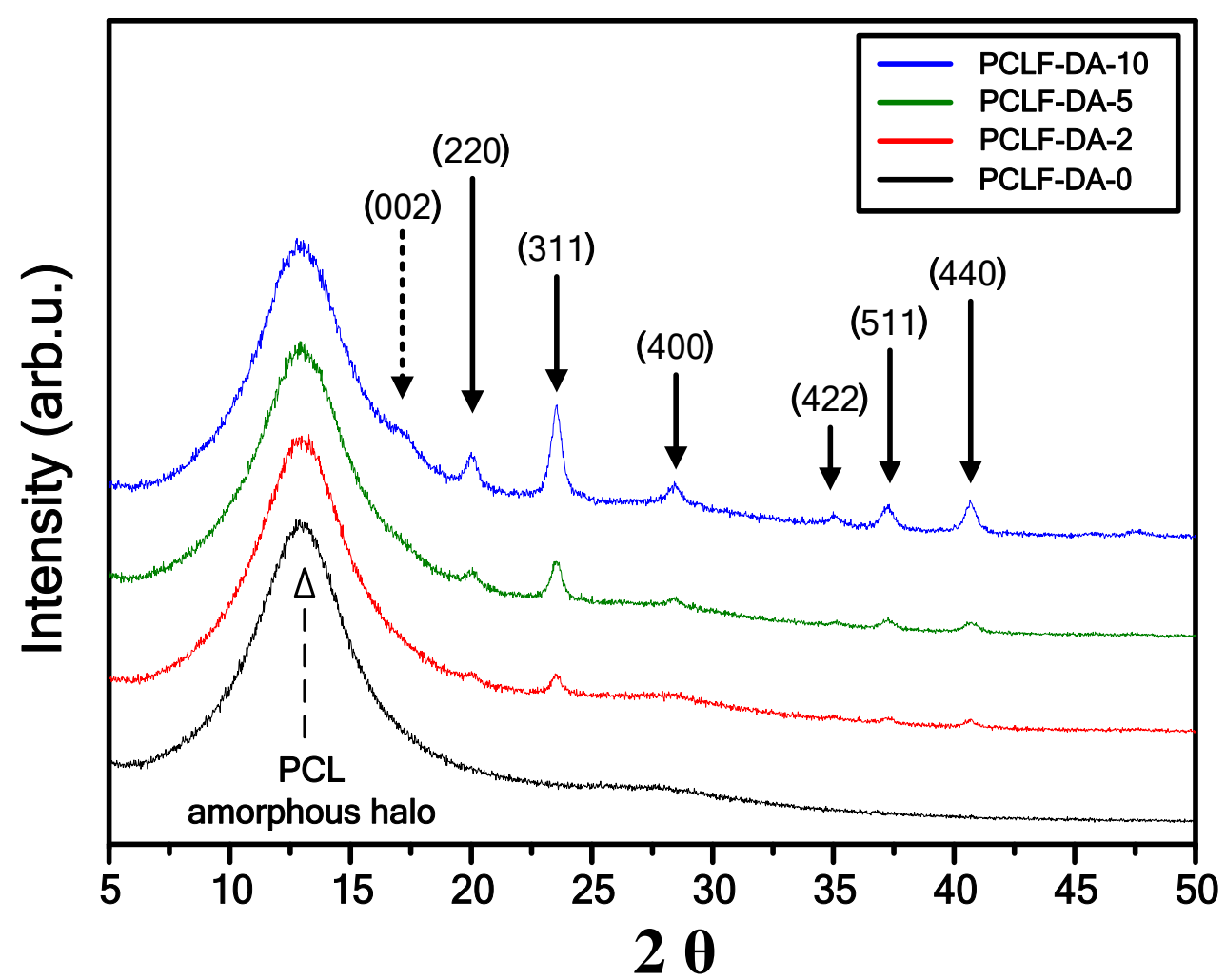

Figure 7. Wide-angle X-ray scattering (WAXS) spectra of the crosslinked PCLF/BMI/IONPs-MWCNTs hybrid samples. The solid arrows indicate the (220), (311), (400), (422), (511), and (440) crystalline reflections of the IONPs. Additionally, the dotted arrow and dashed hollow arrow indicate the (002) crystallographic plane of MWCNTs and amorphous halo of PCL, respectively.

On the basis of the combined results of the TEM and WAXS analyses, a detailed investigation for the incorporation of IONPs-MWCNTs into the PCLF based polymeric matrix can now be constructed. In order to systematically study this novel crosslinked hybrid system, the thermal properties were further characterized. Figure 8 shows the TGA thermograms of the crosslinked PCLF/BMI/IONPs-MWCNTs hybrids with different compositions of IONPs-MWCNTs. The 5\% weight loss temperature of PCLF-DA-0 showed a higher value than those of the other three samples since the presence of carboxylic acid functional groups on the IONPs-MWCNTs decreased the thermal stability of the hybrid samples [46]. Additionally, the char residue at $600{ }^{\circ} \mathrm{C}$ slightly increased with the addition of IONPs-MWCNTs, demonstrating the successful incorporation of the inorganic nanomaterials. Additionally, PCLF-DA-2, PCLF-DA-5, and PCLF-DA-10 hybrids all showed a 5\% weight loss temperature higher than $325^{\circ} \mathrm{C}$. The weight loss temperatures and char yields of these samples are listed in Table 3. The retro-DA reaction temperatures of the hybrid samples were further analyzed by DSC measurements. As shown in Figure 9, the DSC curve of the PCLF-DA-0 sample showed a broad endothermic signal at around $140{ }^{\circ} \mathrm{C}$ because of the cleavage of covalent bonds composed of furan groups of PCLF and maleimide groups of BMI via a retro-DA reaction. For the PCLF-DA-2, PCLF-DA-5, and PCLF-DA-10 hybrids, their DSC thermograms all exhibited a broad endothermic peak, which could be attributed to the combined signal from furan/maleimide, MWCNT/furan, and MWCNT/maleimide retro-DA reactions. From the significant retro-DA signals, it could be expected that these crosslinked PCLF/BMI/IONPs-MWCNTs hybrid system exhibited effective self-repairing ability imparted from the intrinsic thermal reversibility of the DA click chemistry. The retro-DA onset temperatures and heat flows of these hybrid samples were further calculated and tabulated in Table 3. Comparisons between the DSC thermograms of these measured samples showed no significant changes in the onset temperature. It could be further observed that the retro-DA heat flow of the hybrid system was progressively increased with increasing the IONPs-MWCNTs 
fraction, indicating that the introduction of inorganic IONPs-MWCNTs into the composites significantly increased the reactive diene and dienophile sites for DA reaction, and thus enhanced the crosslink density of the DA network. Therefore, the increased heat flow to debond the DA linkages was required. Additionally, no visible endothermic signal related to the melting point of PCL could be observed for the crosslinked PCLF based samples, indicating that the DA crosslinked structure could strongly reduce the mobility of the PCL segments and restrict the molecular chains to further crystallize, consistent with the scattering features of the WAXS results.

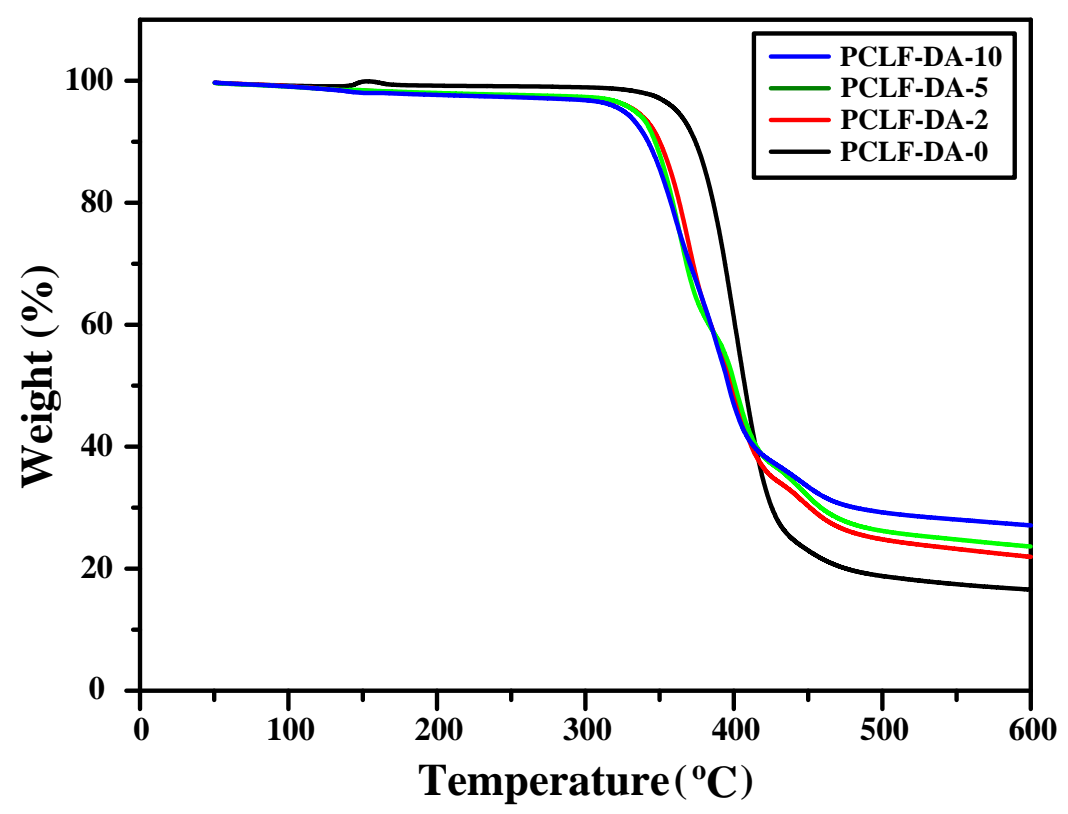

Figure 8. Thermogravimetric analysis (TGA) curves of the crosslinked PCLF/BMI/IONPs-MWCNTs hybrid samples with different loadings of IONPs-MWCNTs.

Table 3. Thermal properties of crosslinked PCLF/BMI/IONPs-MWCNTs hybrid samples.

\begin{tabular}{ccccc}
\hline Sample $^{\mathbf{1}}$ & $\begin{array}{c}\mathbf{5} \mathbf{w t} \mathbf{\%}^{\text {Loss }} \\
\left.\text { Temperature }^{\circ}{ }^{\circ} \mathbf{C}\right)^{\mathbf{2}}\end{array}$ & $\begin{array}{c}\text { Char Yield at } \\
\mathbf{6 0 0}{ }^{\circ} \mathbf{C}(\mathbf{\%})^{\mathbf{3}}\end{array}$ & $\begin{array}{c}\text { Retro-DA Onset } \\
\text { Temperature }\left({ }^{\circ} \mathbf{C}\right)^{\mathbf{4}}\end{array}$ & $\begin{array}{c}\text { Retro-DA Heat } \\
\text { Flow }(\mathbf{J} / \mathbf{g})^{\mathbf{5}}\end{array}$ \\
\hline PCLF-DA-0 & 362 & 16.6 & 82 & 20.5 \\
PCLF-DA-2 & 334 & 21.9 & 81 & 26.5 \\
PCLF-DA-5 & 333 & 23.6 & 83 & 28.3 \\
PCLF-DA-10 & 326 & 27.1 & 82 & 32.5 \\
\hline
\end{tabular}

${ }^{1}$ Designation of crosslinked PCLF/BMI/IONPs-MWCNTs hybrid materials. ${ }^{2,3} 5 \mathrm{wt} \%$ loss temperature and char yield at $600{ }^{\circ} \mathrm{C}$ were measured from TGA analyses. ${ }^{4,5}$ Retro-DA onset temperature and heat flow were measured from DSC analyses.

The magnetic properties of the crosslinked PCLF/BMI/IONPs-MWCNTs hybrid system at room temperature were analyzed by using a vibrating sample magnetometer with an applied field of $-10,000$ Oe $\sim 10,000$ Oe. Shown in Figure 10 are the magnetization curves of the hybrid samples plotted as magnetic moment verses magnetic field. The insert in Figure 10 further shows the detail profiles within the field range of \pm 50 Oe. For the PCLF-DA-2 sample, its magnetization hysteresis loop revealed an S-like curve and exhibited low magnetic coercivity and remanence. Upon increasing the IONPs-MWCNTs loading, both the PCLF-DA-5 and PCLF-DA-10 samples exhibited similar hysteresis loops as that of PCLF-DA-2. Therefore, these magnetic trends clearly indicated that this crosslinked PCLF/BMI/IONPs-MWCNTs hybrid system exhibited a superparamagnetic behavior and there was almost no remanent magnetization while removing the applied magnetic field. Additionally, the magnetization of the PCLF-DA-2, PCLF-DA-5 and PCLF-DA-10 samples estimated from the extreme points (-10,000 Oe and 10,000 Oe) were $700 \mathrm{memu} / \mathrm{g}, 1500 \mathrm{memu} / \mathrm{g}$, and $2800 \mathrm{memu} / \mathrm{g}$, respectively. In comparison with the pristine IONPs, the crosslinked PCLF/BMI/IONPs-MWCNTs samples revealed 
much lower magnetization values because of the low content of IONPs-MWCNTs in the hybrid system and the surface coverage of IONPs-MWCNTs by the non-electromagnetic PCLF polymeric layer.

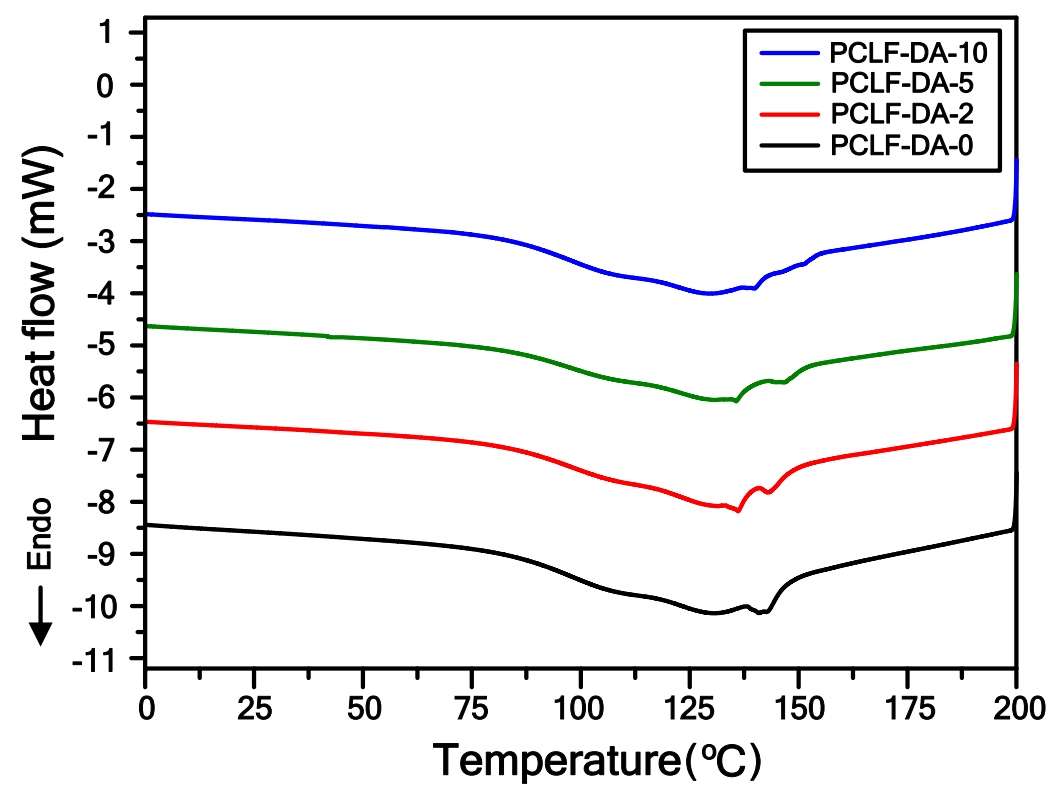

Figure 9. Differential scanning calorimetry (DSC) heating traces of the crosslinked PCLF/BMI/IONPs-MWCNTs hybrid samples with different amounts of IONPs-MWCNTs incorporated. The endothermic enthalpy signals indicate the occurrence of retro-DA reactions of the DA crosslinked system with increasing temperature.

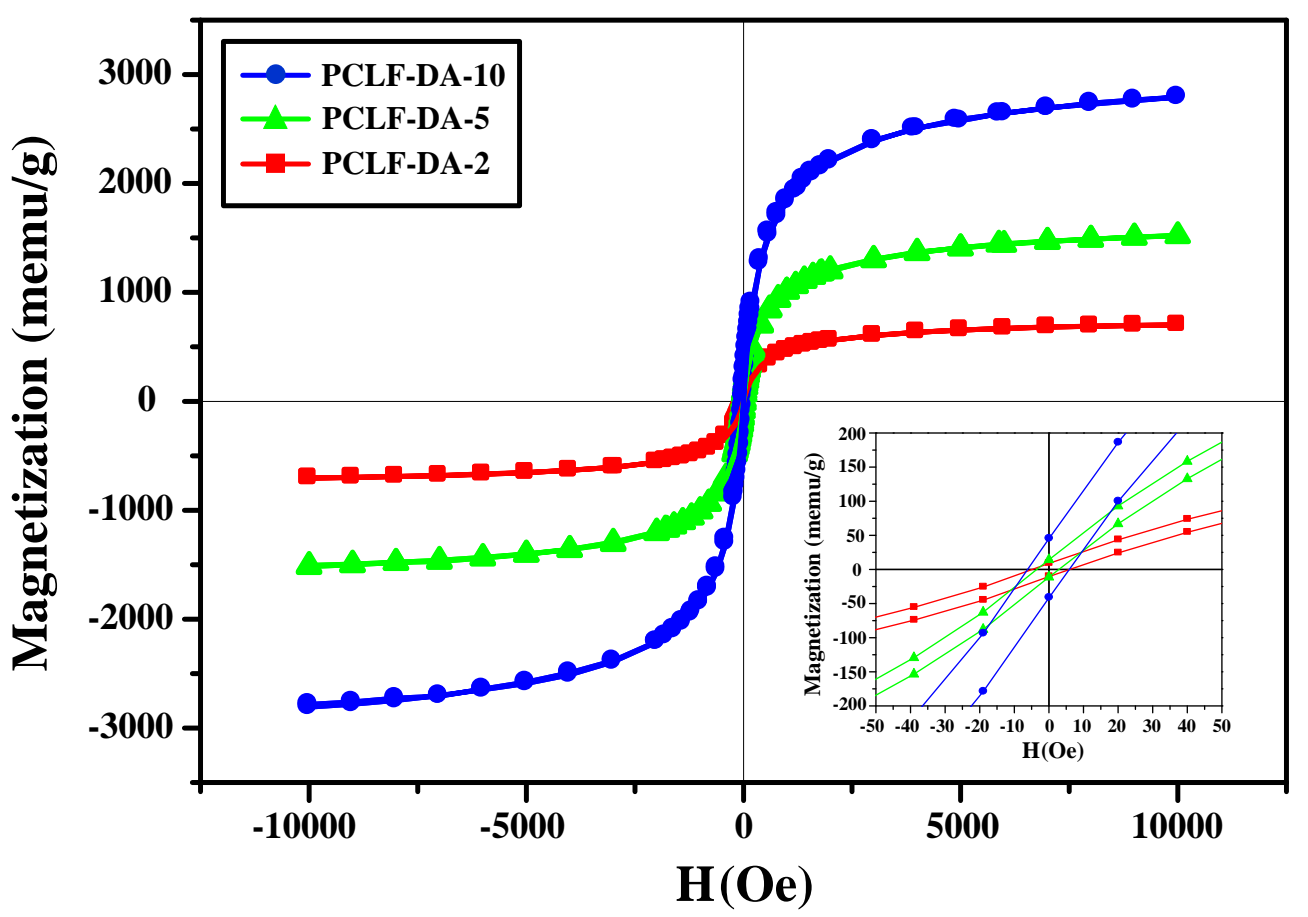

Figure 10. Hysteresis plots of magnetization vs magnetic field for PCLF-DA-2, PCLF-DA-5, and PCLF-DA-10 crosslinked hybrid samples. The insert figure shows the detail profiles within the field range of \pm 50 Oe.

For the crosslinked PCLF/BMI/IONPs-MWCNTs hybrid system, its thermal reversibility related to the DA network was also investigated in solution medium (60 wt \% in DMSO). Figure 11 shows 
the phase transformations observed from the investigations. At low temperature, the crosslinked hybrid only displayed slight swelling even after $24 \mathrm{~h}$, indicating the robust structure supported by the crosslinked DA network. As the hybrid sample was heated to a temperature higher than $140^{\circ} \mathrm{C}$, it could dissolve in the solvent medium, and a solution with good liquidity could be observed. This result clearly demonstrated the occurrence of a retro-DA reaction that disconnected the DA linkages and liberated the molecular chains and inorganic substances from the original network to a plastic state. As the sample was cooled down to a low temperature, a sol-gel transformation could be observed, indicating sufficient recovery of the crosslinked network via a DA reaction.
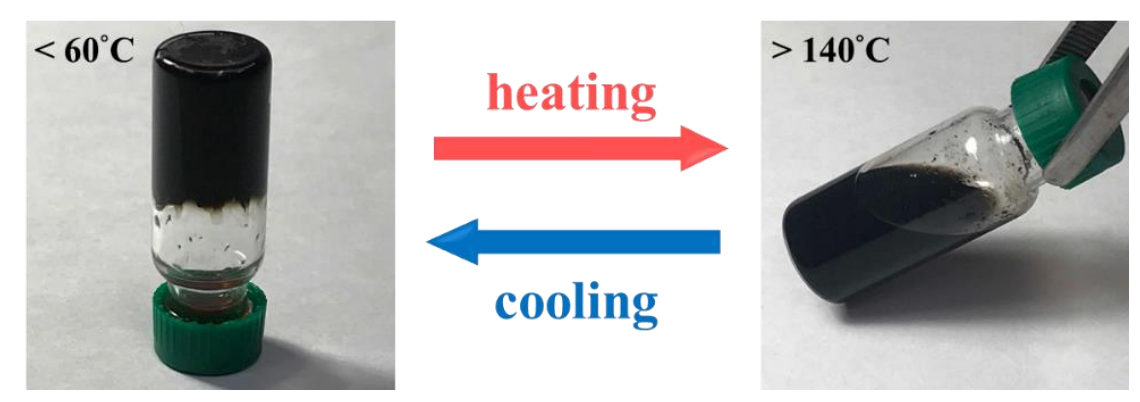

Figure 11. The thermal reversible sol-gel transition of PCLF-DA-5 in DMSO via DA and retro-DA reactions.

To further examine the self-healable performance of the crosslinked PCLF/BMI/IONPs-MWCNTs hybrid system, the structural evolution of a cracked sample with a cut of $5 \mathrm{~mm}$ was monitored under different temperatures using an optical microscopy equipped with a heating stage. From the micrographs shown in Figure 12a-d, it could be observed that the crack was still presented without any change as the hybrid sample was thermally treated at a temperature below $120{ }^{\circ} \mathrm{C}$. Upon increasing the heating temperature to $130{ }^{\circ} \mathrm{C}$, the crack became more flatter, indicating the occurrence of a retro-DA reaction that converted the original DA crosslinked structure to a plastic state. This thermal reversible process strongly increased the chain mobility of the polymeric materials and thus led to the diffusion of polymer chains toward the crack site to heal the structural defect. When the self-healing temperature was further increased to $140{ }^{\circ} \mathrm{C}$, the crack was completely closed within only a few minutes, showing that the PCLF/BMI/IONPs-MWCNTs hybrid system possessed superior self-healing properties. Furthermore, SEM was used to investigate the crack closure at a higher magnification. Figure 13a,b shows the crack evolution before and after the self-healing process, respectively. These micrographs clearly revealed efficient crack closure, consistent with the results obtained from optical microscopy measurements. 

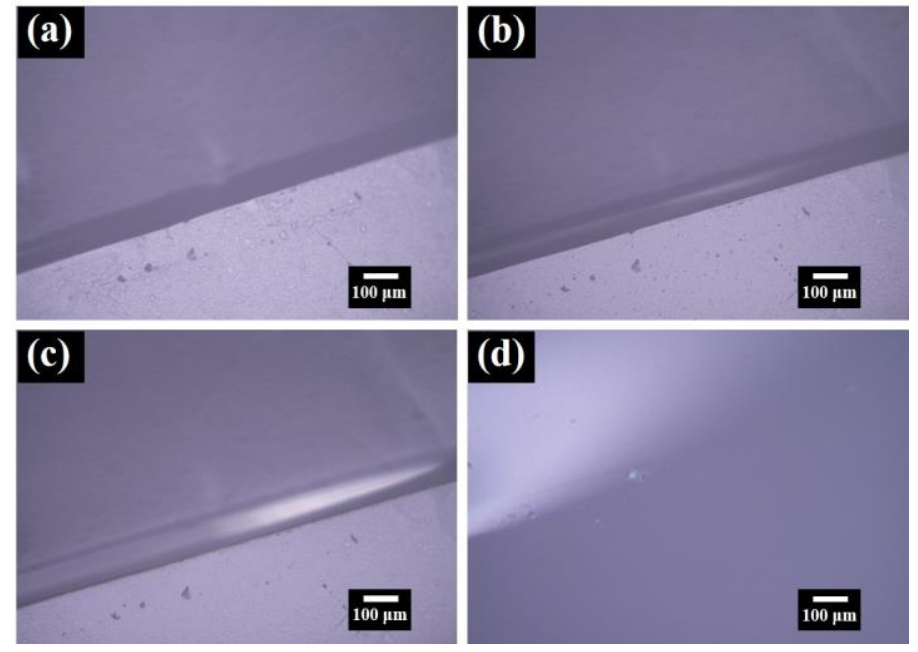

Figure 12. The optical microscope images of PCLF-DA-10 hybrid sample with a crack at (a) room temperature, (b) $120^{\circ} \mathrm{C}$, (c) $130{ }^{\circ} \mathrm{C}$, and (d) $140{ }^{\circ} \mathrm{C}$, respectively.
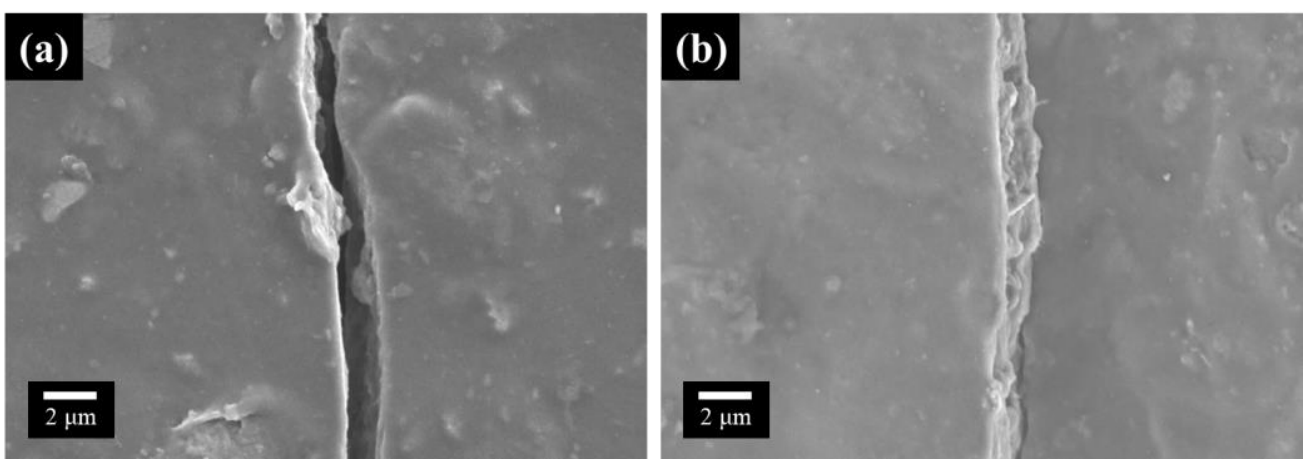

Figure 13. The SEM micrographs of PCLF-DA-10 hybrid sample with a crack (a) before and (b) after the self-healing process at $140{ }^{\circ} \mathrm{C}$.

\section{Conclusions}

In the current study, we have synthesized a PCLF copolymer and further used this functional polymer to develop a novel crosslinked PCLF/BMI/IONPs-MWCNTs hybrid system. The furfuryl-functionalized PCLF copolymer and BMI reagent with maleimide group offered reactive diene and dienophile sites for incorporating IONPs-MWCNTs into the polymeric matrix, therefore inducing a DA crosslinked hybrid network with well-dispersed IONPs-MWCNTs. For the hybrid samples, their hysteresis loops showed S-like curves and revealed no significant remanent magnetization or coercivity, indicating a significant superparamagnetic feature. The self-repairing ability of the magnetic hybrid system was further proven by the scratch healing test. From optical microscopy and SEM observations, we show that the defects gradually healed at a heating temperature above $130{ }^{\circ} \mathrm{C}$, providing evidence that the hybrid system had superior self-healing properties. Thus, we believe that this novel self-healing hybrid system has strong potential for use in future electromagnetic applications.

Author Contributions: Y.-H.L. and W.-J.C. conceived and designed the experiments; Y.-N.Z., M.-F.W. and W.-C.K. performed the experiments; Y.-H.L. analyzed the data and wrote the paper; H.-T.W., T.-F.W. and S.-P.R. contributed reagents, materials, and analysis tools.

Funding: This research received no external funding.

Acknowledgments: The authors gratefully acknowledge the funding from University System of Taipei Joint Research Program (Project No. USTP-NTUT-TMU-107-06).

Conflicts of Interest: The authors declare no conflict of interest. 


\section{References}

1. Toohey, K.S.; Sottos, N.R.; Lewis, J.A.; Moore, J.S.; White, S.R. Self-healing materials with microvascular networks. Nat. Mater. 2007, 6, 581. [CrossRef] [PubMed]

2. Toohey, K.S.; Hansen, C.J.; Lewis, J.A.; White, S.R.; Sottos, N.R. Delivery of two-part self-healing chemistry via microvascular networks. Adv. Funct. Mater. 2009, 19, 1399-1405. [CrossRef]

3. White, S.R.; Sottos, N.R.; Geubelle, P.H.; Moore, J.S.; Kessler, M.R.; Sriram, S.R.; Brown, E.N.; Viswanatha, S. Autonomic healing of polymer composites. Nature 2001, 409, 794-797. [CrossRef] [PubMed]

4. Rule, J.D.; Brown, E.N.; Sottos, N.R.; White, S.R.; Moore, J.S. Wax-protected catalyst microspheres for efficient self-healing materials. Adv. Mater. 2005, 17, 205-208. [CrossRef]

5. Lanzara, G.; Yoon, Y.; Liu, H.; Peng, S.; Lee, W.I. Carbon nanotube reservoirs for self-healing materials. Nanotechnology 2009, 20, 335704. [CrossRef] [PubMed]

6. Shchukin, D.G.; Möhwald, H. Self-repairing coatings containing active nanoreservoirs. Small 2007, 3, $926-943$. [CrossRef] [PubMed]

7. Wu, D.Y.; Meure, S.; Solomon, D. Self-healing polymeric materials: A review of recent developments. Prog. Polym. Sci. 2008, 33, 479-522. [CrossRef]

8. Syrett, J.A.; Becer, C.R.; Haddleton, D.M. Self-healing and self-mendable polymers. Polym. Chem. 2010, 1, 978-987. [CrossRef]

9. Bekas, D.G.; Tsirka, K.; Baltzis, D.; Paipetis, A.S. Self-healing materials: A review of advances in materials, evaluation, characterization and monitoring techniques. Compos. Part B 2016, 87, 92-119. [CrossRef]

10. Chung, C.-M.; Roh, Y.-S.; Cho, S.-Y.; Kim, J.-G. Crack healing in polymeric materials via photochemical [2+2] cycloaddition. Chem. Mater. 2004, 16, 3982-3984. [CrossRef]

11. Scott, T.F.; Schneider, A.D.; Cook, W.D.; Bowman, C.N. Photoinduced plasticity in cross-linked polymers. Science 2005, 308, 1615-1617. [CrossRef] [PubMed]

12. Gruendling, T.; Kaupp, M.; Blinco, J.P.; Barner-Kowollik, C. Photoinduced conjugation of dithioester- and trithiocarbonate-functional RAFT polymers with Alkenes. Macromolecules 2010, 44, 166-174. [CrossRef]

13. Holten-Andersen, N.; Harrington, M.J.; Birkedal, H.; Lee, B.P.; Messersmith, P.B.; Lee, K.Y.C.; Waite, J.H. $\mathrm{pH}$-induced metal-ligand cross-links inspired by mussel yield self-healing polymer networks with near-covalent elastic moduli. Proc. Natl. Acad. Sci. USA 2011, 108, 2651-2655. [CrossRef] [PubMed]

14. Ghosh, B.; Urban, M.W. Self-Repairing Oxetane-substituted chitosan polyurethane Networks. Science 2009, 323, 1458-1460. [CrossRef]

15. Kakuta, T.; Takashima, Y.; Nakahata, M.; Otsubo, M.; Yamaguchi, H.; Harada, A. Preorganized hydrogel: Self-healing properties of supramolecular hydrogels formed by polymerization of host-guest monomers that contain cyclodextrins and hydrophobic guest groups. Adv. Mater. 2013, 25, 2849-2853. [CrossRef] [PubMed]

16. Nakahata, M.; Takashima, Y.; Yamaguchi, H.; Harada, A. Redox-responsive self-healing materials formed from host-guest polymers. Nat. Commun. 2011, 2, 511. [CrossRef] [PubMed]

17. Liu, Y.-L.; Chuo, T.-W. Self-healing polymers based on thermally reversible Diels-Alder chemistry. Polym. Chem. 2013, 4, 2194-2205. [CrossRef]

18. Diels, O.; Alder, K. Synthesen in der hydroaromatischen Reihe. Justus Liebigs Annalender Chemie 1928, 460, 98-122. [CrossRef]

19. Ripoll, J.L.; Rouessac, A.; Rouessac, F. Applications recentes de la reaction de retro-diels-alder en synthese organique. Tetrahedron 1978, 34, 19-40. [CrossRef]

20. Szalai, M.L.; McGrath, D.V.; Wheeler, D.R.; Zifer, T.; McElhanon, J.R. Dendrimers Based on Thermally Reversible Furan-Maleimide Diels-Alder Adducts. Macromolecules 2007, 40, 818-823. [CrossRef]

21. Robertson, A.; Philp, D.; Spencer, N. Recognition-induced control of Diels-Alder cycloaddition. Tetrahedron 1999, 55, 11365-11384. [CrossRef]

22. McElhanon, J.R.; Wheeler, D.R. Thermally responsive dendrons and dendrimers based on reversible furan-maleimide Diels-Alder adducts. Org. Lett. 2001, 3, 2681-2683. [CrossRef] [PubMed]

23. Chujo, Y.; Sada, K.; Saegusa, T. Reversible gelation of polyoxazoline by means of Diels-Alder reaction. Macromolecules 1990, 23, 2636-2641. [CrossRef]

24. Mallek, H.; Jegat, C.; Mignard, N.; Abid, M.; Abid, S.; Taha, M. Reversibly crosslinked self-healing PCL-based networks. J. Appl. Polym. Sci. 2013, 129, 954-964. [CrossRef] 
25. Defize, T.; Thomassin, J.-M.; Alexandre, M.; Gilbert, B.; Riva, R.; Jérôme, C. Comprehensive study of the thermo-reversibility of Diels-Alder based PCL polymer networks. Polymer 2016, 84, 234-242. [CrossRef]

26. Thakur, V.K.; Kessler, M.R. Self-healing polymer nanocomposite materials: A review. Polymer 2015, 69, 369-383. [CrossRef]

27. Arslan, M.; Tasdelen, M.A. Polymer nanocomposites via click chemistry reactions. Polymers 2017, 9, 499-522. [CrossRef]

28. Behera, P.K.; Mondal, P.; Singha, N.K. Self-healable and ultrahydrophobic polyurethane-POSS hybrids by Diels-Alder "click" reaction: A new class of coating material. Macromolecules 2018, 51, 4770-4781. [CrossRef]

29. Wu, S.; Li, J.; Zhang, G.; Yao, Y.; Li, G.; Sun, R.; Wong, C.-P. Ultrafastly self-healing nanocomposites via infrared laser and its application in flexible electronics. ACS Appl. Mater. Interfaces 2017, 9, 3040-3049. [CrossRef]

30. Menon, A.V.; Madras, G.; Bose, S. Ultrafast self-healable interfaces in polyurethane nanocomposites designed using Diels-Alder "Click" as an efficient microwave absorber. ACS Omega 2018, 3, 1137-1146. [CrossRef]

31. Schäfer, S.; Kickelbick, G. Diels-Alder reactions on surface-modified magnetite/maghemite nanoparticles: Application in self-healing nanocomposites. ACS Appl. Nano Mater. 2018, 1, 2640-2652. [CrossRef]

32. Schäfer, S.; Kickelbick, G. Double reversible networks: Improvement of self-healing in hybrid materials via combination of Diels-Alder cross-linking and hydrogen bonds. Macromolecules 2018, 51, 6099-6110. [CrossRef]

33. Baughman, R.H.; Zakhidov, A.A.; Heer, W.A. Carbon nanotubes-The route toward applications. Science 2002, 297, 787-792. [CrossRef] [PubMed]

34. De Volder, M.F.L.; Tawfick, S.H.; Baughman, R.H.; Hart, A.J. Carbon nanotubes: Present and futurecommercial applications. Science 2013, 339, 535-539. [CrossRef] [PubMed]

35. Utsumi, S.; Urita, K.; Kanoh, H.; Yudasaka, M.; Suenaga, K.; Iijima, S.; Kaneko, K. Preparing a magnetically responsive single-wall carbon nanohorn colloid by anchoring magnetite nanoparticles. J. Phys. Chem. B 2006, 110, 7165-7170. [CrossRef] [PubMed]

36. Eder, D. Carbon nanotube-inorganic hybrids. Chem. Rev. 2010, 110, 1348-1385. [CrossRef] [PubMed]

37. Qu, S.; Wang, J.; Kong, J.; Yang, P.; Chen, G. Magnetic loading of carbon nanotube/nano-Fe ${ }_{3} \mathrm{O}_{4}$ composite for electrochemical sensing. Talanta 2007, 71, 1096-1102. [CrossRef]

38. He, Y.; Huang, L.; Cai, J.-S.; Zheng, X.-M.; Sun, S.-G. Structure and electrochemical performance of nanostructured $\mathrm{Fe} 3 \mathrm{O} 4 /$ carbon nanotube composites as anodes for lithium ion batteries. Electrochimica Acta 2010, 55, 1140-1144. [CrossRef]

39. Pawar, S.P.; Marathe, D.A.; Pattabhi, K.; Bose, S. Electromagnetic interference shielding through MWNT grafted $\mathrm{Fe}_{3} \mathrm{O}_{4}$ nanoparticles in PC/SAN blends. J. Mater. Chem. A 2015, 3, 656-669. [CrossRef]

40. Chen, Y.-H.; Huang, Z.-H.; Lu, M.-M.; Cao, W.-Q.; Yuan, J.; Zhang, D.-Q.; Cao, M.-S. 3D Fe ${ }_{3} \mathrm{O}_{4}$ nanocrystals decorating carbon nanotubes to tune electromagnetic properties and enhance microwave absorption capacity. J. Mater. Chem. A 2015, 3, 12621-12625. [CrossRef]

41. Zhong, W.; Liu, P.; Tang, Z.; Wu, X.; Qiu, J. Facile approach for superparamagnetic $\mathrm{CNT}^{-} \mathrm{Fe}_{3} \mathrm{O}_{4} /$ polystyrene tricomponent nanocomposite via synergetic dispersion. Ind. Eng. Chem. Res. 2012, 51, 12017-12024. [CrossRef]

42. Yu, L.; Yang, X.; Ye, Y.; Wang, D. Efficient removal of atrazine in water with a $\mathrm{Fe}_{3} \mathrm{O}_{4} / \mathrm{MWCNTs}$ nanocomposite as a heterogeneous Fenton-like catalyst. RSC Adv. 2015, 5, 46059-46066. [CrossRef]

43. Hekmatara, H.; Seifi, M.; Forooraghi, K. Microwave absorption property of aligned MWCNT/Fe $\mathrm{O}_{4}$. J. Magn. Magn. Mater. 2013, 346, 186-191. [CrossRef]

44. Pramanik, N.B.; Singha, N.K. Direct functionalization of multi-walled carbon nanotubes (MWCNTs) via grafting of poly(furfuryl methacrylate) using Diels-Alder "click chemistry" and its thermoreversibility. RSC Adv. 2015, 5, 94321-94327. [CrossRef]

45. Chang, C.-M.; Liu, Y.-L. Functionalization of multi-walled carbon nanotubes with furan and maleimide compounds through Diels-Alder cycloaddition. Carbon 2009, 47, 3041-3049. [CrossRef]

46. Fan, W.; Zuo, L.; Zhang, Y.; Chen, Y.; Liu, T. Mechanically strong polyimide/carbon nanotube composite aerogels with controllable porous structure. Compos. Sci. Technol. 2018, 156, 186-191. [CrossRef]

(C) 2019 by the authors. Licensee MDPI, Basel, Switzerland. This article is an open access article distributed under the terms and conditions of the Creative Commons Attribution (CC BY) license (http:/ / creativecommons.org/licenses/by/4.0/). 